\title{
Respiratory function in the morbidly obese before and after weight loss
}

\author{
P S THOMAS, E R T C OWEN, G HULANDS, J S MILLEDGE \\ From the Departments of Respiratory Medicine, Surgery and Anaesthesia, Northwick Park Hospital and \\ Clinical Research Centre, Harrow
}

ABSTRACT The morbidly obese are known to have impaired respiratory function. A prospective study of the changes in lung volumes, carbon monoxide transfer, and arterial blood gas tensions was undertaken in 29 morbidly obese patients before and after surgery to induce weight loss. Before surgery the predominant abnormality in respiratory function was a reduction in lung volumes. These increased towards normal predicted values after weight loss, with significant increases in functional residual capacity, residual volume, total lung capacity, and expiratory reserve volume. The increases $₫$ ranged from $14 \%$ for total lung capacity to $54 \%$ for expiratory reserve volume. After weight loss had been induced the smokers showed mild hyperinflation and air trapping. Resting arterial blood gas tensions improved, with a rise in arterial oxygen tension from 10.63 to $13.02 \mathrm{kPa}$ and a fall in arterial carbon dioxide tension from 5.20 to $4.64 \mathrm{kPa}$. There was no correlation between weight loss and the changes in blood gas tensions or lung volumes. Loss of weight in the morbidly obese is thus associated with improved lung function. The effects of smoking on lung function could be detected after weight loss, but were masked before treatment by the opposing effects of obesity on residual volume and functional residual capacity.

\section{Introduction}

The morbidly obese often have respiratory ailments ranging from mild breathlessness on exercise to the Pickwickian syndrome of daytime somnolence, hypoxaemia, polycythaemia, and cor pulmonale' or obstructive sleep apnoea. ${ }^{2}$ Because cigarette smoking reduces appetite many obese subjects smoke and this will also have a deleterious effect on respiratory function.

Bariatric surgery (surgery for obesity) has been reserved for obesity refractory to dietary measures. Operations previously performed, such as jejunoileal and gastric bypass procedures, have been associated with important complications and mortality. Vertical banded gastroplasty (fig 1 ) is a simpler operation with a low morbidity and a mortality of $0.3 \%$ in one study. ${ }^{3}$ It has become the most popular of the vertical

Address for reprint requests: Dr J S Milledge, Department of Respiratory Medicine, Northwick Park Hospital, Harrow, HA1 3UJ.

Accepted 28 February 1989

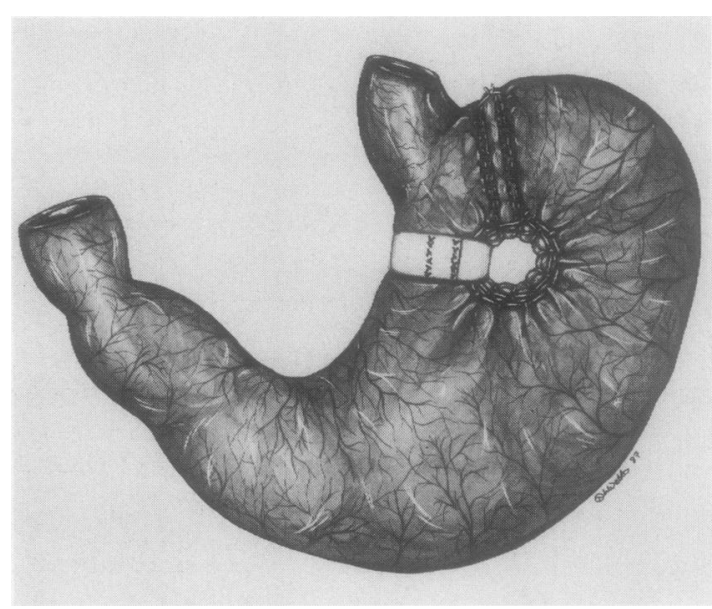

Fig 1 Vertical banded gastroplasty. The stomach is partitioned by a double vertical staple line and the outlet of the resulting pouch is controlled by an inelastic band. (From Flejou et al"; reproduced by courtesy of the "British Journal of Surgery".) 


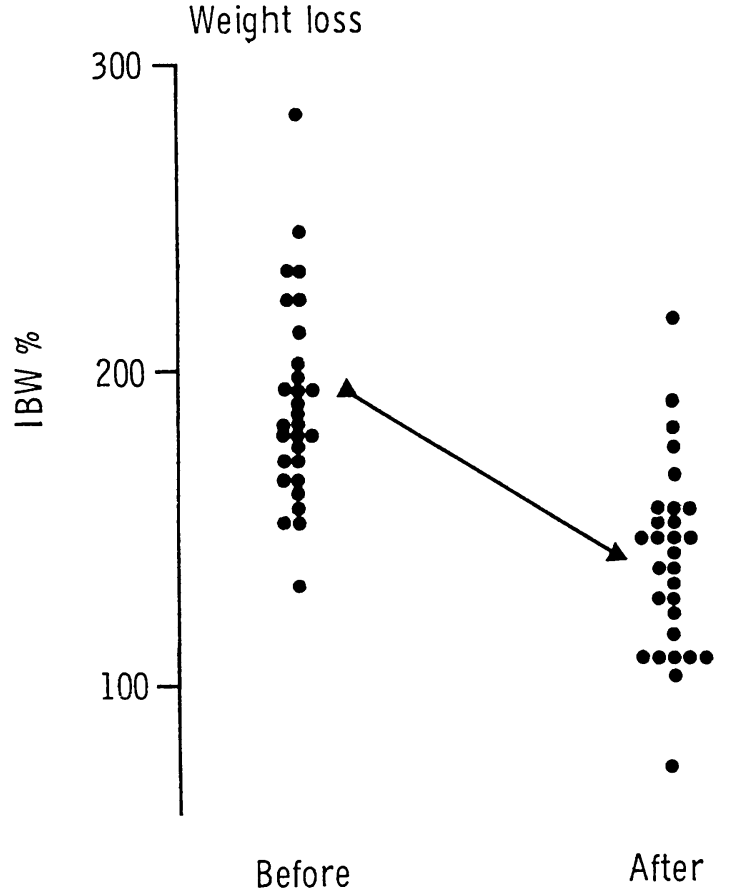

Fig 2 Weight distribution of patients before and after surgery and weight loss. $\triangle$ Mean value. IBW-ideal body weight.

gastroplasties and is currently the most widely performed bariatric operation worldwide. ${ }^{3}$

\section{Patients and methods}

We studied 29 consecutive subjects (one male) undergoing bariatric surgery. Their mean age was 39.3 (range 18-56) years. Fourteen either were current smokers or had smoked in the past. All were selected by virtue of being $100 \%$ or more above their ideal body weight or $50 \mathrm{~kg}$ or more above for at least three years. ${ }^{4}$ All the subjects had been given a supervised outpatient diet for one year and had failed to lose weight. Informed consent to the investigations and operation was obtained from all patients. Their mean weight before operation was 126.4 (range 92-174) kg. The body mass index was calculated as weight divided by the square of the height $\left(\mathrm{kg} / \mathrm{m}^{2}\right){ }^{4}$

Forced expiratory volume in one second $\left(\mathrm{FEV}_{1}\right)$ and forced vital capacity (FVC) were measured by dry wedge spirometry (Vitalograph) with the patients seated after 15 minutes' rest. Peak expiratory flow (PEF) was measured by a Wright's peak flow meter. Lung volumes (total lung capacity (TLC), functional residual capacity (FRC), residual volume (RV), and expiratory reserve volume (ERV)) were measured by
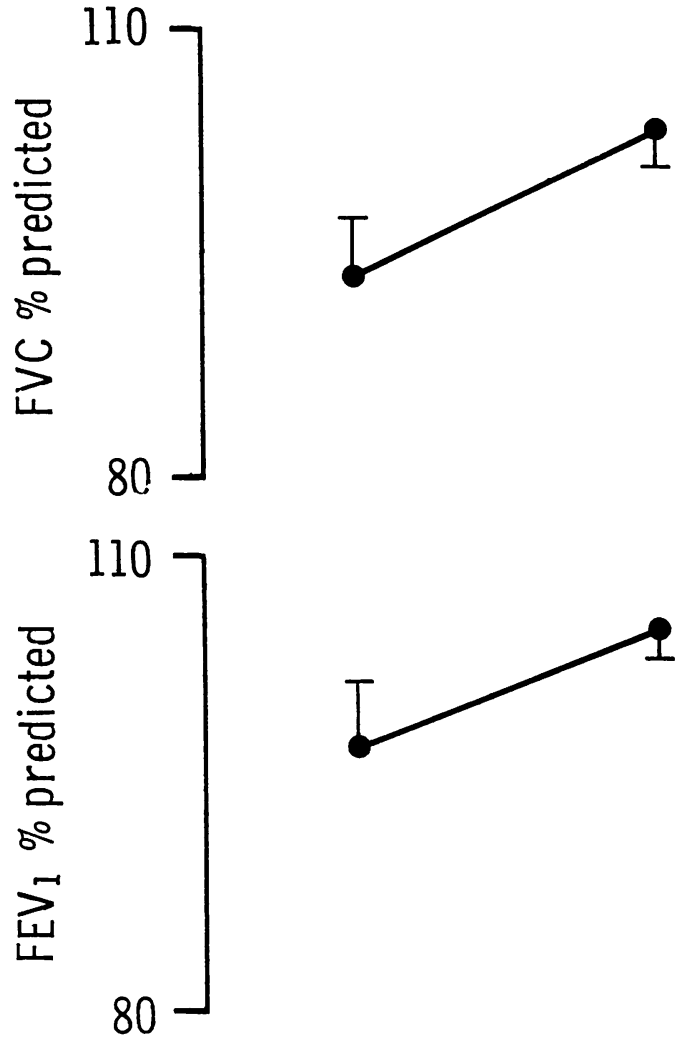

Before

After

Fig 3 Mean FEV and forced vital capacity $(F V C)$, before and after weight loss. Bar lines indicate 1 SEM.

helium dilution. Carbon monoxide gas transfer (TLCO) was assessed by the single breath technique in 10 subjects. The Krogh factor (KCO) was calculated as the TLCO per litre of effective alveolar volume. Arterial blood gases were obtained from the radial artery in 14 patients with a local anaesthetic, with the subject seated and breathing air after resting for 15 minutes.

Results have been expressed as percentages of predicted values. ${ }^{5}$ The regression equations that determine predicted values do not take weight into account except in the case of ERV. ${ }^{5}$ Because with the weight correction many patients would have had a negative predicted ERV, we expressed ERV as percentage predicted on the basis of ideal body weight.

Statistical analysis was by paired two tailed $t$ test unless otherwise stated.

\section{Results}

The subjects were followed up for a mean of 26 (range 


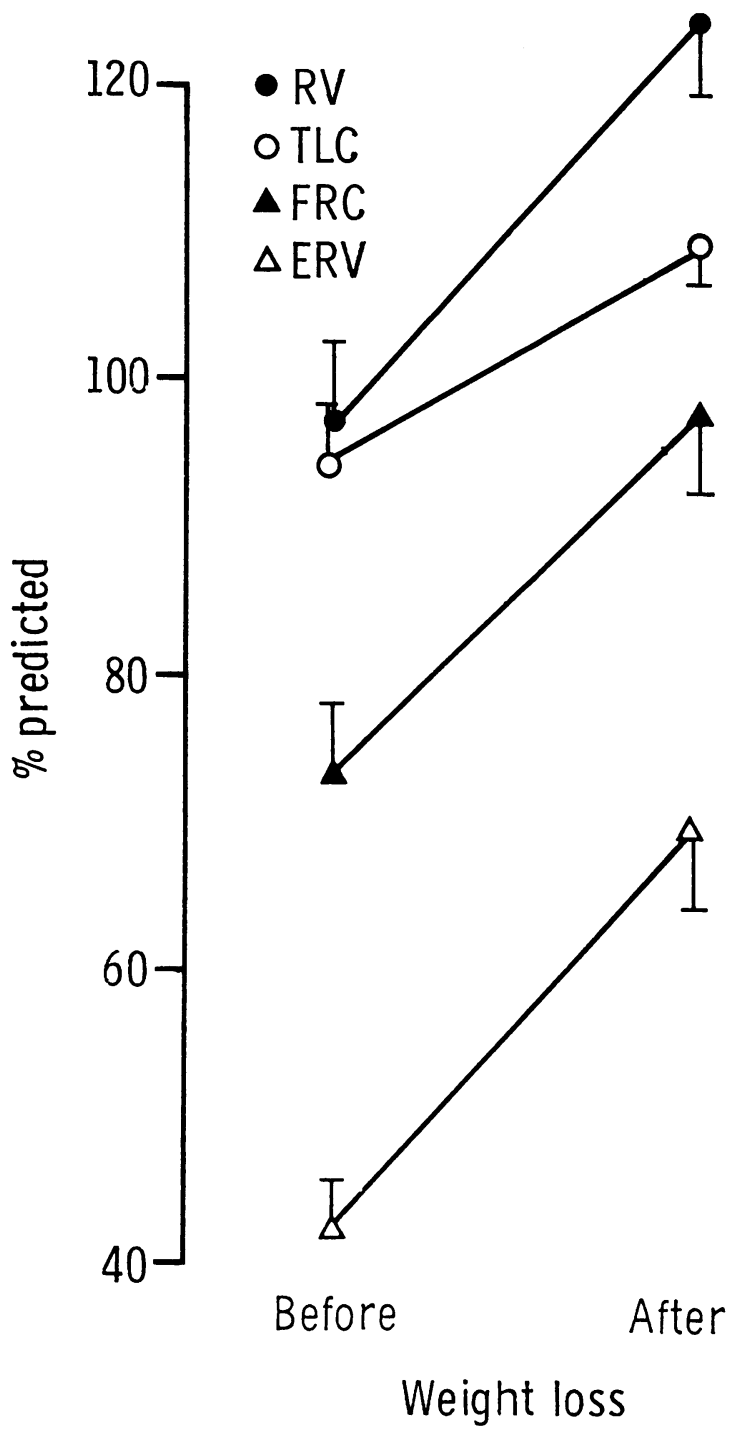

Fig 4 Mean residual volume ( $R V)$, total lung capacity (TLC), functional residual capacity, and expiratory reserve volume before and after weight loss. Bar lines indicate I SEM.

2-66) months. All lost weight-mean 34.2 (range 264) $\mathrm{kg}$ (fig 2). The main changes in dynamic and static lung volumes are summarised in figures 3 and 4 . Before weight loss FVC, FEV , and PEF were not significantly below predicted values; after weight loss, however, there was a small but significant rise in FVC of $7 \%(p=0.002)$ and in $F_{E V}$ of $6 \%(p=0.017)$, with no change in PEF.

FRC, TLC, RV, and ERV were all reduced before surgery, especially ERV (figs 3 and 4). All showed a significant increase after weight loss. When values for the whole group were expressed as change in \% predicted values from preoperative values $\mathrm{RV} \frac{\bar{\sigma}}{\bar{\omega}}$ increased by $26 \%$, TLC by $14 \%$, FRC by $37 \%$, and $\vec{\nabla}$ ERV by $54 \%$. Similar changes were seen in smokers and non-smokers. There was no difference in lung is function between smokers and non-smokers before $\vec{\circ}$ surgery. After surgery and weight loss, however, FRC, TLC, and RV (but not ERV) were larger than predicted in smokers and significantly greater than in those who had never smoked ( $\mathrm{p}<0.05$, unpaired $t$ test).

Arterial blood gas analysis showed an initial slight hypoxaemia, with a mean arterial oxygen tension $\left(\mathrm{PaO}_{2}\right)$ of 10.6 (SEM 1.64) kPa, which improved after weight loss to normal values $(13.0(0.55) \mathrm{kPa}, \mathrm{p}<$ 0.005: fig 5). Arterial carbon dioxide tension $\left(\mathrm{PCO}_{2}\right)$ fell from $5.2(0.6)$ to $4.6(0.16) \mathrm{kPa}(\mathrm{p}<0.05)$ after surgery, suggesting an improvement in either ventilation or gas exchange. There was, however, no significant change in TLCO or Kco in the 16 subjects who had the measurements made before and after surgery. Mean (SEM) TLCO was $8.32(0.93) \mathrm{mm} \mathrm{min}^{-1} \mathrm{kPa}^{-1}$ before and $8.12(0.67)$ after weight loss; Kco was 1.83 $(0.1)$ before and $1.76(0.07) \mathrm{mmol} \mathrm{min}{ }^{-1} \mathrm{kPa}^{-1} l^{-1}$ after weight loss.

Improvement in blood gas tensions and lung function variables did not correlate with change in weight, whether actual weight loss, percentage ideal body weight change, or change in body mass index or weight loss index.

Tables of the full data are available (from JSM).

\section{Discussion}

The benefits of loss of weight on lung function in the morbidly obese are seen clearly in this study of 29 patients. There was a significant improvement in lung $\mathrm{O}$ function, most notably a $54 \%$ increase in ERV and improvement in gas exchange.

Physicians interpreting lung function results should bear in mind obesity as a cause of a decrease in lung volumes or hypoxaemia, and a case may be made for noting weight routinely when patients are assessed in the laboratory.

Obesity probably causes respiratory embarrassment by several mechanisms. Our study has emphasised that ERV, RV, FRC, and TLC are reduced by comparison $\stackrel{\mathcal{P}}{+}$ with values after weight loss (fig 4), most of the change $\square$ in TLC being accounted for by a change in ERV and $\bar{O}$

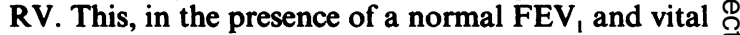
capacity, suggests that splinting of the diaphragm by $\mathbb{D}$ intra-abdominal fat may prevent its full excursion, and $\sigma$ this would account for some of the changes. It has been suggested that pulmonary compliance is decreased by 


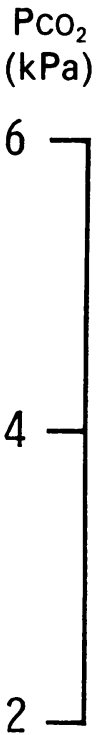

Before

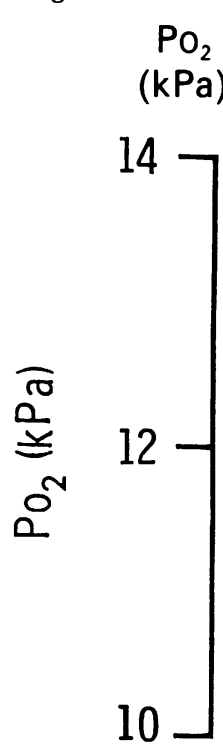

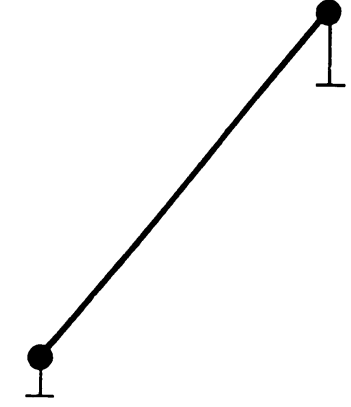

Before

Fig 5 Mean arterial carbon dioxide and oxygen tensions $\left(\mathrm{PCO}_{2}\right.$ and $\left.\mathrm{PO}_{2}\right)$ before and after surgery.

the deposition of subcutaneous adipose tissue, acting as a fat envelope, in the chest wall. ${ }^{6}$ Respiratory muscle function may also be compromised, both by an initial mechanical disadvantage caused by fat and by the need to use more energy to expand the lungs than is used by a lean individual. ${ }^{7}$ Gas exchange deficits are evident from our study. This may be due to a combination of ventilation-perfusion mismatch and hypoventilation. Causes of ventilation-perfusion mismatch include underventilation of well perfused lower lung regions, which has been observed in obesity. ${ }^{89}$ Airway closure was detected by the nitrogen washout method in some obese patients during tidal breathing. This was reversed by weight loss and an increase in the FRC. ${ }^{10}$

The fact that carbon monoxide uptake did not change after weight loss suggests that ventilationperfusion mismatch was less important than hypoventilation in causing the abnormal gas exchange. This is supported by observations of hypoventilation and disordered breathing patterns in the obese. ${ }^{2}$ The abnormal breathing pattern may be due to an abnormality of central control or possibly obese individuals reach the limit of the amount of work that can be achieved by the respiratory muscles. If a central abnormality were present an attenuated ventilatory response to carbon dioxide and to hypoxia would be expected. In a study of four obese patients before and after weight loss there was a fall in the slope of ventilatory response to carbon dioxide when weight was lost, the reverse of what would be expected. "Thus the available data favour the idea that ventilation is limited by the increased work of breathing in obesity, relative hypoventilation occurring because of respiratory muscle fatigue and chronic inability to respond to hypercapnia and hypoxia.?

Most previous studies of the effect of bariatric operations have shown an improvement in arterial oxygenation only in a relatively few patients. Other workers, like us, have been unable to show a convincing correlation between loss of weight and improvement of lung function variables. In one study that purported to show a positive relation between reduction in shunt and weight loss only six of 11 patients showed improved oxygenation and loss of weight. ${ }^{12}$ The relation between weight loss and improvements in measured lung function variables is not clear. Our results have too much scatter to show a definite link. Perhaps a different index, such as pulmonary compliance or a measurement of respiratory muscle fatigue, might relate more directly to weight loss.

Before surgery there was no difference in spirometric values and lung volumes between smokers and non-smokers, whereas after weight loss smokers were found to have significantly more air trapping than the non-smokers, who had reached normal predicted respiratory values. The effect of smoking on lung function tends to be obscured by morbid obesity.

Patients with this degree of obesity have a high risk of postoperative complications, but vertical banded gastroplasty results in less metabolic disturbance than alternative operations. In this series no patient died in the postoperative period, though two patients died at 
home of pulmonary embolism six and seven weeks after their operation.

Vertical banded gastroplasty has been carried out in this hospital in 60 patients, including the 29 reported here. Major wound infection has occurred in six cases and minor infection (reddening and serous discharge) in 17. Other complications, such as thromboembolism ( 1 case), chest infection (8), and gastric bleeding (1), added to the morbidity and late complications included the two deaths from pulmonary embolism, staple line dehiscence (4 cases), stoma stenosis (5), and regaining of weight (4). The life expectancy of morbidly obese people is considerably reduced, a trend that may be reversed by bariatric surgery. With better respiratory function after weight loss these patients can look forward to increased mobility.

\section{References}

1 Burwell CS, Robin ED, Whaley RD, Bickelmann AG. Extreme obesity associated with alveolar hypoventilation: a Pickwickian syndrome. Am J Med 1956;21: 811-8.

2 Walsh RE, Michaelson ED, Harkleroad LE, Zighelboim A, Sackner MA. Upper airway obstruction in obese patients with sleep disturbance and somnolence. Ann Intern Med 1972;76:185-92.

3 Mason EE. Vertical banded gastroplasty for obesity. Arch Surg 1982;117:701-6.
4 National Institutes of Health. Consensus development conference on surgical treatment of morbid obesity. Ann Surg 1979;189:455-7.

5 Cotes JE. Lung function: assessment and application in medicine. 4th ed. Oxford: Blackwell, 1979.

6 Naimark A, Cherniack RM. Compliance of the respiratory system and its components in health and obesity. J Appl Physiol 1960;15:377-82.

7 Rochester DF, Enson Y. Current concepts in the pathogenesis of the obesity-hypoventilation syndrome. Am J Med 1974;57:402-20.

8 Holley HS, Milic-Emili J, Becklake MR, Bates DV. Regional distribution of pulmonary ventilation and perfusion in obesity. $J$ Clin Invest 1967;46:475-81.

9 Barrera F, Hillyer P, Ascandio G, Betchtel J. The distribution of ventilation, diffusion and blood flow in obese patients with normal and abnormal blood gases. Am Rev Respir Dis 1973;108:819-30.

10 Santesson J, Nordenstrom J. Pulmonary function in extreme obesity. Influence of weight loss following intestinal shunt operation. Acta Chir Scand 1978;suppl 482:36-40.

11 Sugarman HJ, Fairman RP, Baron PL, Kwentos JA. Gastric surgery for respiratory insufficiency of obesity. Chest 1986;89:81-6.

12 Vaughan RW, Cork RC, Hollander D. The effect of massive weight loss on arterial oxygenation and pulmonary function tests. Anesthesiology 1981;54:325-8.

13 Flejou J-F, Owen ERTC, Smith AC, Price AB. Effect of vertical banded gastroplasty on the natural history of gastritis in patients with morbid obesity: a follow-up study. Br J Surg 1988;75:705-7. 